\title{
Propiedad familiar, control y efecto generación y RSC
}

\author{
Family ownership, control and generation and CSR \\ Laura Cabeza García $^{\mathrm{a}^{*}} \cdot$ María Sacristán Navarro ${ }^{\mathrm{b}} \cdot$ Silvia Gómez Ansón ${ }^{\mathrm{c}}$ \\ ${ }^{a}$ Departamento de Dirección y Economía de Empresa, Universidad de León (León) \\ ${ }^{b}$ Departamento de Economía de la Empresa (Administración, Dirección y Organización), Universidad Rey Juan \\ Carlos (Madrid) \\ ${ }^{c}$ Departamento de Administración de Empresas, Universidad de Oviedo (Oviedo)
}

D A T OS A R TÍ C U L O

Historial:

Recibido 04-12-2013

Aceptado 05-04-2014

Palabras clave:

RSC

Tansparencia

Propiedad Familiar

Control Familiar

Generación Familiar

Códigos JEL:

M14, G34

\section{A R T I C L E IN F O}

Article history:
Received 04-12-2013
Accepted 05-04-2014

Keywords:

CSR

Disclosure

Family Ownership

Family Control

Family Firms'generation

JEL codes:

M14, G34
RES U M E N

El objetivo de este trabajo es analizar el papel de la propiedad familiar en la política de Responsabilidad Social Corporativa (RSC) medida ésta a partir de varios indicadores de transparencia informativa sobre las acciones sociales y medioambientales realizadas por las empresas. Utilizando una muestra de empresas no financieras españolas en el periodo 2004-2010 e identificando el último gran propietario de la compañía a partir de las cadenas de propiedad, encontramos que el compromiso en RSC es menor en empresas con propiedad familiar, si bien, los resultados no indican que la presencia del fundador de la compañía afecte significativamente a las políticas informativas de la empresa. Por otro lado, los resultados relativos al efecto del control ejercido por las familias sobre la transparencia en RSC son mixtos, sugiriendo que el control familiar influye en la política de dar información o no sobre RSC, pero no en la amplitud de la información suministrada al mercado.

\footnotetext{
A B S T R A C T

This paper aims to analyse the effect of family ownership on firms' Corporate Social Responsibility (CSR) actions measured through several CSR disclosure variables. Using a sample of non-financial Spanish listed firms over the period 2004-2010 and after identifying the ultimate owner of the firms, the results show that family firms are less committed with CSR disclosure policies although the presence of the families' founders does not affect the firms' CSR disclosure. The results also seem to reveal a negative effect of family control on the decision to disclose CSR practices, but not on the amplitude of the information disclosed.
}

\footnotetext{
* Autor de contacto.
}

Correoselectrónicos: laura.cabeza@unileon.es,maria.sacristan@urjc.es,sgomez@uniovi.es 


\section{Introducción}

En las últimas décadas, la maximización del beneficio económico para los accionistas ha dejado paso a otros objetivos más amplios pues, a partir de la Teoría de los stakeholders (Freeman, 1984; Mitchell et al., 1997) se ha puesto de manifiesto la necesidad de que las compañías cubran las expectativas económicas, legales, éticas y discrecionales de todos los grupos de interés, no sólo de los accionistas (Carroll, 1991), así como de informar sobre las acciones realizas en este sentido. Este creciente interés por una gestión de la empresa que atiende las necesidades de sus stakeholders ha contribuido a la consolidación de la Responsabilidad Social Corporativa (RSC) como disciplina de investigación (Crane et al., 2008; Aguinis y Glavas, 2012). Así, a pesar de la no existencia de un concepto unánime de RSC, se podría definir ésta como el conjunto de acciones voluntarias de una empresa, más allá de sus obligaciones económicas y legales, para mejorar así las condiciones sociales y medioambientales en sus negocios y en su interacción con los distintos stakeholders (Comisión Europea, 2002).

Aunque la mayor parte de los estudios empíricos previos sobre RSC se han centrado en la relación entre el desempeño social y el rendimiento económico (véase, por ejemplo, para una revisión, los trabajos de Griffin y Mahon, 1997; Orlitzky et al., 2003; Peloza, 2009), diversos trabajos han tratado de analizar los factores determinantes de la realización de actividades sociales (Moore, 2001; Simpson y Kohers, 2002; Lindgreen et al., 2009; Aguinis y Glavas, 2012; entre otros). A este respecto, en los últimos años se han llevado a cabo estudios empíricos que consideran la estructura de propiedad como un factor explicativo de la RSC. Así, tanto a nivel internacional (Arora y Dharwadkar, 2011; Harjoto y Jo, 2011; Yong et al., 2011, Walls et al., 2012; entre otros) como en el contexto español (Fernández-Sánchez et al., 2011; Godos-Díez et al., 2012), se ha estudiado la influencia del grado de concentración de la propiedad sobre la RSC. De igual forma, en menor medida, en especial para el caso de España, se ha analizado también la influencia de la identidad del principal accionista sobre la realización de acciones de naturaleza social (por ejemplo, Barnea y Rubin, 2010; Harjoto y Jo, 2011; Dam y Scholtens, 2012; McGuire et al., 2012). Distintos estudios, han abordado asimismo la influencia de la identidad de los accionistas sobre la transparencia en RSC (Haniffa y Cooke, 2005; Ghazali, 2007; Kuo et al., 2012; Testera y Cabeza, 2013). Dentro de los distintos tipos de accionistas, la propiedad familiar y su efecto sobre los resultados empresariales (Sharma et al., 2012) ha sido objeto de numerosos estudios. Sin embargo, a nivel internacional no son muchos los trabajos que consideran la naturaleza familiar de la empresa como determinante de la RSC, en especial considerando la identidad del último propietario siguiendo las cadenas de propiedad.

Nuestro trabajo, ante esta carencia de estudios a nivel internacional sobre este tema, y también, para el caso particular de España, explora esta cuestión utilizando para ello una muestra de empresas no financieras españolas en el periodo 2004-2010. Nos planteamos diferentes preguntas: ¿Hasta qué punto es diferente la política en RSC de las empresas familiares frente a las no familiares? ¿Es diferente la política de RSC en las empresas familiares en función de si la familia además controla los órganos de gobierno? ¿Influye la generación de la familia en el compromiso de las empresas con las acciones de naturaleza social?

El resto del artículo se ha estructurado como sigue: la Sección 2 se dedica a la revisión de la literatura relacionada con el tema objeto de estudio; la muestra, las variables utilizadas y la metodología se describen en la Sección 3. En la cuarta Sección se presentan los resultados de los análisis y, finalmente, en la Sección 5 se exponen las principales conclusiones del trabajo.

\section{Revisión de la literatura}

El desarrollo de la Teoría de los stakeholders (Freeman, 1984; Mitchell et al., 1997; Agle et al., 1999) ha puesto de manifiesto la necesidad de considerar a todos los grupos de interés relevantes para la actividad empresarial en la definición de los objetivos empresariales. Así, muchas compañías han tendido a ampliar sus objetivos más allá de su rendimiento económico incorporando su desempeño social y medioambiental. Este creciente interés por una gestión de la empresa que atiende las necesidades de sus stakeholders ha contribuido a la consolidación de la RSC, es decir, las acciones voluntarias tomadas por las compañías respecto a sus empleados, a la sociedad y al medioambiente (Barnea y Rubin, 2010).

En este sentido, un posible factor a considerar a la hora de explicar las acciones de RSC emprendidas por la empresa es su estructura de propiedad al influir ésta en las decisiones y actuaciones empresariales (Ortiz de Mandojana et al., 2011). En este sentido, la literatura incluye argumentos que 
avalan tanto una relación positiva como negativa entre el grado de concentración de la propiedad empresarial y la realización de acciones de RSC.

Entre los argumentos a favor una relación positiva entre concentración de propiedad y acciones relacionadas con RSC se cuentan que en aquellas compañías con una estructura de propiedad más concentrada, la $\mathrm{RSC}$, puede tener más peso en la toma de decisiones para evitar el coste de ser calificada como una empresa "socialmente irresponsable" (Barnea y Rubin, 2010); el que considerando que los grandes accionistas pueden tener un objetivo distinto al rendimiento a corto plazo asociado a sus inversiones (Blair, 1995; Monks y Minow, 1995), las empresas que cuenten con una propiedad más concentrada dispondrán de un abanico más amplio de posibles inversiones $\mathrm{y}$, entre ellas, tendrán especial cabida las de mayor horizonte temporal (Hoopes y Miller, 2006), como por ejemplo, inversiones en RSC (Mahapatra, 1984; Johnson y Greening, 1999); el hecho de que la realización de actividades de naturaleza social puede hacer más fácil la obtención de fondos así como la reducción de costosas sanciones derivadas de decisiones judiciales (Graves y Waddock, 1994; Consolandi et al., 2008); o que la presencia de grandes accionistas puede disuadir a los directivos de reducir la inversión en RSC al objeto de disponer de más recursos para perseguir sus propios objetivos a corto plazo a expensas del resto de stakeholders (Consolandi et al., 2008).

No obstante, también es posible plantear una relación entre concentración de propiedad y acciones en RSC en sentido contrario. Así, la existencia de una propiedad concentrada en manos de accionistas significativos puede llevar a una gestión empresarial encaminada hacia la obtención de beneficios privados, que puede ir en perjuicio de otros grupos de interés (Johnson et al., 2000; Cuervo, 2004). En concreto, los propietarios dominantes pueden disfrutar de un poder excesivo que favorezca la expropiación de recursos a los minoritarios a fin de maximizar sus propios beneficios privados, reduciendo así el compromiso de la empresa con la RSC (Brammer y Pavelin, 2008). Asimismo, teniendo en cuenta el horizonte temporal de la toma de decisiones, también es posible que existan inversores que pueden invertir y desinvertir con facilidad y que sólo se centren en objetivos a corto plazo, con independencia del volumen de recursos comprometidos en una compañía (Porter, 1992).

Sin embargo, a la hora de analizar qué tipo de actividades de RSC llevan a cabo las empresas o en qué medida lo hacen es necesario considerar que el papel desempeñado por los grandes accionistas no solo depende de su participación en la propiedad, sino también de su identidad o su naturaleza. Así, la dirección o el equipo directivo de una compañía puede tener una percepción distinta sobre la importancia de ser social y ambientalmente responsable en función de las expectativas de los diferentes propietarios en términos del beneficio económico y del desempeño social y ambiental (Ndemanga y Koffi, 2009). Por ejemplo, generalizando las denominaciones que Bushee (1998) y Dikolli et al. (2009) emplean refiriéndose a los inversores institucionales, se puede plantear que la participación significativa de inversores miopes o transitorios en el capital de una compañía presionará para que la gestión empresarial se encamine hacia los resultados cortoplacistas, reduciendo así el desarrollo de acciones de RSC.

En lo relativo a la empresa familiar, ésta se caracteriza por un horizonte de gestión a largo plazo, lo que influye en el interés por su supervivencia y en su compromiso con una mejora en la reputación de la compañía (Anderson et al., 2003; McVey y Draho, 2005). Siguiendo este argumento, cabría esperar que las empresas familiares tiendan a estar más comprometidas con la reputación corporativa, sean más proclives a involucrase en actividades altruistas para reforzar su ego (Schulze et al., 2003) y a invertir en acciones de RSC (Déniz y Cabrera, 2005) en beneficio de todos los stakeholders. Las familias o inversores individuales pueden perseguir así objetivos no sólo de naturaleza económica (Sharma et al., 1997; Anderson y Reeb, 2003) distintos a los de los inversores anónimos que buscan una alta rentabilidad (LaPorta et al., 1999; Miller y Le Breton-Miller, 2006). La empresa familiar puede estar interesada en mantener una buena reputación y las acciones de RSC pueden ser una forma de conseguirlo (Bachiller et al., 2013). Otra posible explicación que apoyaría la mayor implicación de las empresas familiares en RSC es la orientación relacional $\mathrm{y}$ colectivista de una compañía familiar a la hora de relacionarse con los otros grupos de interés, frente a una orientación más individualista en el caso de las empresas no familiares. Es decir, a pesar del riesgo asociado a las inversiones en actividades de RSC, una familia puede estar dispuesta a asumirlo, a cambio del mantenimiento de su riqueza socio-emocional y de una buena reputación (Gómez-Mejía et al., 2007). Es cierto que las empresas no familiares también pueden perseguir estos objetivos no económicos $\mathrm{y}$ beneficios en su reputación vinculados a la 
realización de acciones de naturaleza social (Bear et al., 2010), sin embargo, la superposición entre la familia y la empresa convertiría estas motivaciones especialmente relevantes en el caso de las compañías familiares (Habberson et al., 2003; Sharma, 2008).

En línea con los argumentos anteriores, Graafland (2002) concluyó que las empresas familiares holandesas, sobre todo las de gran tamaño, muestran un mayor interés por la RSC que las empresas no familiares. Por su parte, en el trabajo de Berrone et al. (2010) para una muestra de compañías americanas se constata que, al margen de cualquier consideración política o económica, cuando la preservación de la riqueza socioemocional de la empresa tiene prioridad, como ocurre con los propietarios familiares, es más probable que la compañía participe en el cumplimiento social. Los resultados de Jo y Harjoto (2011) también para EEUU sugieren igualmente una relación positiva entre las empresas familiares y la realización de acciones de RSC. En el caso concreto de la transparencia en RSC, Prado-Lorenzo et al. (2009) para una muestra de empresas españolas no financieras cotizadas encuentran que la presencia de un accionista significativo que ejerza control sobre la compañía y que sea un individuo aumentará la probabilidad de que la empresa siga las directrices del Global Reporting Iniciative (GRI) como modelo a la hora de dar información sobre sus actividades de naturaleza social y medioambiental.

Por el contrario, también es posible argumentar una relación negativa entre propiedad familiar y RSC. Una familia que tenga invertida en una compañía una parte importante de su riqueza puede quizás estar más interesada en el retorno de sus inversiones, es decir, en recibir una ganancia económica que compense el riesgo de la inversión realizada y que garantice la viabilidad de la empresa para las futuras generaciones familiares $y$, en consecuencia, no se preocupará tanto por las cuestiones ambientales y sociales. Además, tal y como ponen de manifiesto Déniz y Cabrera (2005) para el caso español, la mayoría de las empresas familiares no piensan que la RSC sea una fuente de ventaja competitiva pero sí que ellas disponen de los recursos y capacidades necesarias para realizar acciones sociales. Se trataría de un perfil filantrópico respecto a la RSC, ya que las empresas familiares considerarían que deben mantener una amplia relación con la sociedad a pesar de que es percibida como un coste. Otro grupo de empresas considera que ni la realización de actividades sociales es una fuente de ventaja competitiva ni que ellas tengan los recursos necesarios para hacer este tipo de actividades.

En esta línea, Ndemanga y Koffi (2009) encontraron que aquellas compañías en las cuales el mayor porcentaje de participación y derecho de voto está en manos de una familia son menos transparentes sobre sus prácticas de RSC. De igual forma, los trabajos de López-Iturriaga y López-deForonda (2011) y de Dam y Scholtens (2012) para una muestra de empresas europeas sugieren una relación negativa entre la presencia de una familia o individuos en el capital de la compañía como principal accionista y la realización de acciones de RSC. Por su parte, McGuire et al. (2012) para EEUU utilizan un índice de rentabilidad social y encuentran una relación negativa entre el estatus de ser empresa familiar y una pobre rentabilidad social. Sin embargo, no encuentran evidencia de que el gobierno corporativo esté relacionado con la rentabilidad social.

En resumen, de acuerdo con los fundamentos teóricos anteriores, la naturaleza familiar de la empresa parece incidir en la realización de acciones de RSC, aunque los resultados de los estudios empíricos no son siempre concluyentes en el signo de la relación. En este contexto, a continuación, utilizando una muestra de empresas españolas se lleva a cabo un análisis de carácter descriptivo para profundizar en la relación anterior, teniendo en cuenta además otras variables no frecuentemente consideradas, como el control familiar o la generación de la empresa, a la hora de analizar los determinantes de las prácticas de RSC en la empresa familiar.

\section{Muestra, variables y metodología}

\subsection{Muestra}

La base de datos del estudio está compuesta por el conjunto de empresas españolas no financieras cotizadas en el IGBM en el periodo 2004-2010, un total de 128 compañías (744 observaciones). Las empresas financieras, de seguros y de servicios de inversión no fueron consideradas debido a sus especiales características. De esta base de datos inicial hemos excluido las empresas filiales, las observaciones correspondientes al año de fusión de una empresa o aquellos casos donde no había toda la información disponible debido a problemas financieros de la compañía (2 empresas, 11 observaciones). 
Tras aplicar estos filtros, la muestra final se compone de 126 empresas españolas cotizadas que se corresponden con 733 observaciones de las cuales 454 son observaciones de empresas familiares (EF), es decir, compañías controladas por una familia o individuo que actúa como último propietario (siguiendo la metodología de La Porta et al., 1999; Claessens et al., 2000, 2002; Faccio y Lang, 2002). Así, siempre que una familia es el mayor propietario aparente o el último propietario de una empresa, y esa propiedad excede del 10\%, consideramos a la compañía como empresa familiar. Por ello buscamos las participaciones que poseían todos los individuos o miembros de una familia y las hemos sumado. Los miembros familiares se han identificado siguiendo los apellidos, es decir, hemos considerado relaciones familiares de sangre o bien hemos sumado las participaciones en caso de matrimonio.

La información relativa a la estructura de propiedad la obtuvimos principalmente de los Informes Anuales de Gobierno Corporativo depositados en la Comisión Nacional del Mercado de Valores (CNMV).

\subsection{Variables}

Medidas de RSC. La complejidad inherente al concepto de RSC ha derivado en distintas formas de medir esta variable: cuestionarios, indicadores de transparencia a partir de la información de los informes anuales o memoria de $\mathrm{RSC}$, o ratings de organizaciones especializadas, entre las más comunes. En este sentido, nuestra variable dependiente son distintos indicadores de la transparencia informativa en RSC a lo largo del periodo 2004-2010: (a) APM es una variable dummy que toma valor 1 si la empresa está adherida al Pacto Mundial y 0 en caso contrario ${ }^{1}$; (b) MEMORIA es una variable dummy que toma valor 1 si la empresa da información social y/o medioambiental en su informe anual o memoria y 0 en caso contrario; (c) GRI es una variable dummy que toma valor 1 si la empresa elabora un informe de RSC o de sostenibilidad de acuerdo con las recomendaciones del GRI y 0 en caso contrario $^{2}$; (d)

\footnotetext{
${ }^{1}$ Esta variable puede ser un indicador de transparencia porque se trata de informar de lo que se hace, pero también una acción de RSC como tal porque se supone que informa de una evolución en las prácticas que están llevando a cabo de acuerdo con los principios del Pacto Mundial.

${ }^{2}$ Global Reporting Iniciative (GRI) es una organización compuesta por miles de expertos a lo largo del mundo cuyo objetivo es desarrollar un marco de normas de alcance mundial con las pautas para mejorar la producción y la comprensión de información transparente, fiable y
}

MEMORIAGRI1 es una variable dummy que toma valor 1 si la empresa da información social y/o medioambiental en su informe anual o memoria o elabora un informe de RSC o de sostenibilidad de acuerdo con las recomendaciones del GRI y 0 en caso contrario; (e) MEMORIAGRI2 es una variable dummy que toma valor 1 si la empresa da información social $\mathrm{y} / \mathrm{o}$ medioambiental en su informe anual o memoria y elabora un informe de RSC o de sostenibilidad de acuerdo con las recomendaciones del GRI y 0 en caso contrario; (f) un índice global $y$ distintos sub-índices de transparencia informativa en RSC construidos siguiendo las recomendaciones establecidas por el GRI a partir de la información que ofrece cada empresa en las variables definidas en las cuatro dimensiones consideradas: perfil, económica, ambiental y social.

La dimensión Perfil, se compone de 38 ítems o variables sobre los cuales las empresas dan información sobre su estrategia social $\mathrm{y}$ medioambiental, de los detalles de su perfil y de la participación y los compromisos del gobierno corporativo en la organización. La dimensión Económica de la sostenibilidad se refiere a los impactos de la organización sobre las condiciones económicas de sus stakeholders así como en los sistemas económicos en que se desenvuelve (locales, nacionales $\mathrm{y}$, en su caso, internacionales) (6 variables). La dimensión Ambiental de la sostenibilidad se ocupa de los impactos que la empresa puede ocasionar sobre la vida de los sistemas naturales, incluidos los ecosistemas, la tierra, el aire y el agua. Las 30 variables ambientales de esta dimensión están relacionadas con las materias primas procedentes de la naturaleza, los residuos enviados al medioambiente, el compromiso de la organización con la biodiversidad o el cumplimiento de las normas ambientales, entre otras.

Por último, la dimensión Social se divide, a su vez, en otras 4 subcategorías (34 variables). La primera de ellas informa si las prácticas laborales se ajustan a la Declaración de Principios y Derechos Fundamentales del Trabajo. La siguiente subcategoría muestra el respeto de la organización hacia los Derechos Humanos, como por ejemplo, no discriminación, igualdad de género, libertad de asociación, trabajo infantil, mano de obra forzada o los derechos indígenas. En tercer lugar, se considera

comparable de informes de sostenibilidad. Así, su objetivo principal es la divulgación de información social, medioambiental y económica. 
el impacto que la compañía puede tener en la comunidad local en la que opera, cómo gestiona los posibles riesgos que pueden surgir de las interacciones con otras instituciones sociales, en la información sobre los peligros asociados a la corrupción y a los sobornos, a la indebida influencia en la política pública y en lo relativo a prácticas monopolísticas. Finalmente, la subcategoría sobre la Responsabilidad sobre los Productos aborda los aspectos de la cobertura de información en los productos y servicios de una organización y que afectan directamente a sus clientes, tales como el etiquetado, la privacidad del cliente y su satisfacción, posibles riesgos para la salud del consumo de ciertos productos, etc.

Además de las cuatro dimensiones anteriores, se han considerado en la construcción del índice global de transparencia en RSC otras cuatro variables vinculadas a otras recomendaciones del GRI sobre cuestiones más generales relativas a la memoria de sostenibilidad que las empresas elaboran. De esta forma, se analiza si la compañía ofrece información acerca del proceso de elaboración de dicha memoria; si en ella se incluye un informe de conclusiones de un Comité de Expertos independiente a la empresa que les ayude a impulsar la transparencia de la información divulgada; si incluye un informe que justifique su verificación por una entidad independiente a la propia empresa que revise la información relativa a las prácticas ambientales, de seguridad y sociales contenida en la memoria de sostenibilidad; y si hace mención a las perspectivas o retos futuros que pretende realizar en términos de mejora social o medioambiental.

Esta última metodología de construcción de índices y subíndices que enfatiza amplitud -que no extensión- de la información divulgada en relación a los elementos seleccionados ha sido utilizada en estudios previos como Wallance et al. (1994) o Archel (2003). Es necesario además comentar que el GRI establece los principios GRI2 para el periodo de tiempo 2002-2005 y el GRI3 para 2006-2010. Por ello, y dado que nuestro periodo temporal objeto de estudio es 2004-2010, para homogeneizar toda la información nos hemos centrado en aquellas variables que eran comunes entre ambos indicadores (GRI2 y GRI3), un total de 112 sobre 130 incluidas en el GRI3.

Variables explicativas. Las principales variables explicativas son varios indicadores de la naturaleza de la empresa familiar: propiedad, control y generación familiar. Así, definimos una variable dummy que toma valor 1 si la empresa tiene como mayor accionista significativo y/o último accionista significativo a una familia o a un individuo considerando el último propietario y tiene además un porcentaje de participación en el capital superior al $10 \%$ y 0 en caso contrario (EF). FAMCONTROL es una variable dummy que toma valor 1 si algún miembro de la familia es el presidente del consejo de administración o el máximo directivo (Chief Executive Officer -CEO-) y 0 en caso contrario. FUNDADOR es una variable dummy que toma valor 1 si la empresa es de propiedad familiar en manos del fundador y 0 en caso contrario.

\subsection{Metodología}

La metodología utilizada consiste en distintos análisis de estadística descriptiva de diferencias en las sub-muestras de empresas familiares versus no familiares; de control familiar versus no control familiar dentro de la sub-muestra de empresas familiares y con presencia del fundador versus no fundador dentro igualmente de la sub-muestra de empresas familiares: test chi-cuadrado para las variables dicotómicas y análisis de diferencias de medias y medianas en lo que se refiere a los distintos indicadores del índice de transparencia considerado. En este sentido, tras aplicar el test de KolmogorovSmirnoff se rechaza la hipótesis nula de normalidad de dichas variables. Ello indica que las variables no siguen una distribución normal. Cuando se da esta circunstancia $\mathrm{y}$, por tanto, la distribución de los datos no es simétrica, se considera que la mediana constituye una medida de resumen de la tendencia central más informativa que la media (Campbell, 1974; Freund y Simon, 1994). Debido a ello se recurre a la mediana para describir los datos a analizar y para confirmar si las diferencias detectadas son estadísticamente significativas se aplicó la prueba no paramétrica de la U de MannWhitney para dos muestras independientes.

\section{Resultados}

La Tabla 1 muestra el compromiso en RSC de las empresas medido como la adhesión al Pacto Mundial así como otros indicadores de transparencia en RSC diferenciando entre los dos tipos de compañías consideradas: empresas de propiedad familiar y empresas no familiares. En todas las variables, salvo en la relativa al hecho de proporcionar información social y/o medioambiental en la memoria o informe anual (MEMORIA), las empresas no familiares muestran un valor superior, siendo además las diferencias estadísticamente 
significativas. Así, por ejemplo, un $36,56 \%$ de las empresas no familiares están adheridas al Pacto Mundial (APM) frente al 30,62\% de las empresas familiares (diferencia significativa al 10\%). De igual forma, un $34,41 \%$ de las empresas no familiares elabora un informe de sostenibilidad siguiendo las directrices del Global Reporting Iniciative (GRI), siendo ese porcentaje inferior en el caso de las empresas familiares $(26,43 \%)(p<0,05)$. Además, las empresas no familiares son más transparentes si consideramos conjuntamente la posibilidad de dar información social o medioambiental en su informe anual y al mismo tiempo elaborar un informe según lo establecido por el GRI (MEMORIAGRI2) (28,81\% de las empresas no familiares y un $18,72 \%$ de las familiares) $(\mathrm{p}<0,05)$.

Estos resultados difieren, por ejemplo, de los obtenidos por Jo y Harjoto (2011) para EEUU que encuentran una relación positiva entre la propiedad familiar y la RSC, pero son similares a los señalados en estudios que consideran países europeos de manera individual, como Ndemanga y Koffi (2009) que, para el caso de Suecia, encontraron que aquellas compañías en las cuales el mayor porcentaje de participación y derecho de voto está en manos de una familia son menos transparentes sobre sus prácticas de RSC. De igual forma, los trabajos de López-Iturriaga y López-de-Foronda (2011) y de Dam y Scholtens (2012) para una muestra de empresas europeas sugieren una relación negativa entre la presencia de una familia o individuos en el capital de la compañía como principal accionista y la realización de acciones de RSC. La menor necesidad de financiación externa que caracteriza a las empresas familiares frente a las no familiares, el tradicional secretismo atribuido a las empresas familiares o la preferencia a invertir en acciones que deriven más directamente en un rendimiento económico más visible en lugar de acciones de RSC pueden explicar los resultados obtenidos.

Por otro lado, tal y como muestra la Tabla 2 (Panel A), si además de un criterio de propiedad se considera uno de control por parte de la familia, es decir, si además de ser una empresa familiar algún miembro de la familia es el presidente del consejo de administración o el máximo directivo (FAMCONTROL), los resultados ponen de manifiesto que el control familiar tiene un efecto negativo sobre las medidas de RSC que se refieren a la publicación de información, sin medir el nivel, tipo o cuantía de información suministrada, pues en todos los casos donde las diferencias son significativas los porcentajes de las variables que se refieren a la adhesión al Pacto Mundial o publicación de alguna memoria o información de RSC son siempre menores para las empresas familiares donde además hay control familiar. Por ejemplo, casi el $56 \%$ de las empresas familiares pero sin control familiar dan información social o medioambiental en su memoria o elaboran un informe según las directrices del GRI (MEMORIAGRI1), mientras que esa cifra se sitúa en el $42 \%$ para las empresas familiares y de control familiar $(p<0,01)$. Nuestros resultados son en este sentido similares a los de McGuire et al. (2012) que definiendo la empresa familiar a partir de un criterio de propiedad y control concluyen que las empresas familiares tenían un peor rendimiento social que las no familiares. Las compañías familiares, como las no familiares, poseen varios stakeholders, pero quizás el hecho de ser familiares, especialmente en el caso en el que la propiedad coincida con la

Tabla 1

Comparación del compromiso en RSC según la naturaleza familiar de las empresas: 2004-2010.

\begin{tabular}{llll}
\hline Variable & $\begin{array}{l}\text { Empresa familiar } \\
(\mathrm{n}=454)\end{array}$ & $\begin{array}{l}\text { Empresa no familiar } \\
(\mathrm{n}=279)\end{array}$ & Chi-cuadrado \\
\hline APM & $30,62 \%$ & $36,56 \%$ & $2,765 \dagger$ \\
MEMORIA & $20,04 \%$ & $19,35 \%$ & 0,052 \\
GRI & $26,43 \%$ & $34,41 \%$ & $5,290 *$ \\
MEMORIAGRI1 & $45,81 \%$ & $53,40 \%$ & $3,985^{*}$ \\
MEMORIAGRI2 & $18,72 \%$ & $28,81 \%$ & $5,152^{*}$ \\
\hline$\dagger \mathrm{p}<0,10 ; * \mathrm{p}<0,05 ; * * \mathrm{p}<0,01 ; * * * \mathrm{p}<0,001$ & &
\end{tabular}

APM es una variable dummy que toma valor 1 si la empresa está adherida al Pacto Mundial; MEMORIA es una variable dummy que toma valor 1 si la empresa da información social y/o medioambiental en su informe anual o memoria; GRI es una variable dummy que toma valor 1 si la empresa elabora un informe de RSC o de sostenibilidad de acuerdo con las recomendaciones del GRI; MEMORIAGRI1 es una variable dummy que toma valor 1 si la empresa da información social y/o medioambiental en su informe anual o memoria o elabora un informe de RSC o de sostenibilidad de acuerdo con las recomendaciones del GRI; MEMORIAGRI2 es una variable dummy que toma valor 1 si la empresa da información social y/o medioambiental en su informe anual o memoria y elabora un informe de RSC o de sostenibilidad de acuerdo con las recomendaciones del GRIEn 
Tabla 2

Influencia del control y la generación en el compromiso en RSC de las empresas familiares: 2004-2010.

\begin{tabular}{llll}
\hline \multicolumn{3}{l}{ Panel A: Existencia de control o no familiar por parte de la familia (FAMCONTROL) } \\
\hline & $\begin{array}{l}\text { Control familiar } \\
(\mathrm{n}=319)\end{array}$ & $\begin{array}{l}\text { No control familiar } \\
(\mathrm{n}=135)\end{array}$ & Chi-cuadrado \\
\hline APM & $25,70 \%$ & $42,22 \%$ & $12,182 * * *$ \\
MEMORIA & $21,63 \%$ & $16,30 \%$ & 1,684 \\
GRI & $20,06 \%$ & $41,48 \%$ & $22,379 * * *$ \\
MEMORIAGRI1 & $41,69 \%$ & $55,55 \%$ & $7,343 * *$ \\
MEMORIAGRI2 & $14,73 \%$ & $28,15 \%$ & $11,217^{* *}$ \\
\hline
\end{tabular}

Panel B: Generación de la familia (FUNDADOR)

$\begin{array}{lll}\text { Empresa familiar } & \text { Empresa familiar no } & \text { Chi-cuadrado } \\ \text { fundador }(\mathrm{n}=282) & \text { fundador }(\mathrm{n}=172)\end{array}$

\begin{tabular}{llll} 
APM & $29,43 \%$ & $32,56 \%$ & 0,491 \\
MEMORIA & $18,08 \%$ & $23,26 \%$ & 1,782 \\
GRI & $29,43 \%$ & $21,51 \%$ & $3,447 \dagger$ \\
MEMORIAGRI1 & $47,52 \%$ & $43,02 \%$ & 0,869 \\
MEMORIAGRI2 & $17,73 \%$ & $20,34 \%$ & 0,481 \\
\hline
\end{tabular}

$\mathrm{n}=454 ; \dagger \mathrm{p}<0,10 ; * \mathrm{p}<0,05 ; * * \mathrm{p}<0,01 ; * * * \mathrm{p}<0,00$

Por control familiar entendemos la presencia del grupo propietario familiar en puestos como el presidente del consejo de administración o el máximo ejecutivo de la empresa. Por la generación de la familia consideramos si el fundador se encuentra en el grupo que ostenta la propiedad de la empresa o no.

Tabla 3

Comparación del índice de transparencia GRI según la naturaleza familiar de las empresas: 2004-2010.

\begin{tabular}{lccccccc}
\hline \multirow{2}{*}{ Variable } & \multicolumn{2}{l}{$\begin{array}{l}\text { Empresa familiar } \\
(\mathrm{n}=120)\end{array}$} & \multicolumn{5}{c}{$\begin{array}{l}\text { Empresa no familiar } \\
(\mathrm{n}=96)\end{array}$} \\
\cline { 2 - 8 } & Media & Mediana & $R P^{a}$ & Media & Mediana & $R P^{a}$ & U Mann Whitney \\
\hline ÍNDICE GLOBAL & 0,809 & 0,825 & 90,31 & 0,890 & 0,920 & 131,23 & $3577,5^{* * *}$ \\
- PERFIL & 0,821 & 1 & 98,37 & 0,905 & 1 & 121,16 & $4544,5^{* *}$ \\
- ECONÓMICO & 0,674 & 0,833 & 95,15 & 0,809 & 1 & 125,19 & $4157,5^{* * *}$ \\
- MEDIOAMBIENTE & 0,597 & 0,600 & 90,30 & 0,781 & 0,917 & 131,25 & $3576^{* * *}$ \\
- SOCIAL & 0,597 & 0,632 & 88,53 & 0,778 & 0,912 & 133,46 & $3363,5^{* * *}$ \\
- Laboral y ética & 0,694 & 0,750 & 91,83 & 0,836 & 1 & 129,33 & $3760^{* * *}$ \\
- Derechos humanos & 0,616 & 0,750 & 89,58 & 0,811 & 0,875 & 132,16 & $3489^{* * *}$ \\
- Sociedad & 0,554 & 0,600 & 90,04 & 0,761 & 1 & 131,58 & $3544,5^{* *}$ \\
- Resp. productos & 0,548 & 0,578 & 96,44 & 0,703 & 1 & 123,57 & $4313^{* *}$ \\
\hline
\end{tabular}

${ }^{\mathrm{a}} \mathrm{RP}$ hace referencia al rango promedio de los datos

$\dagger \mathrm{p}<0,10 ; * \mathrm{p}<0,05 ; * * \mathrm{p}<0,01 ; * * * \mathrm{p}<0,001$

INDICE GLOBAL es un índice total en transparencia informativa en RSC construido siguiendo las recomendaciones establecidas por el GRI a partir de la información que ofrece cada empresa en las variables definidas en las cuatro dimensiones consideradas en el GRI: perfil, económica, ambiental y social 
Tabla 4

Influencia del control y la generación en el índice de transparencia GRI de las empresas familiares: 2004-2010.

\begin{tabular}{|c|c|c|c|c|c|c|c|}
\hline \multicolumn{8}{|c|}{ Panel A: Existencia de control familiar por parte de la familia } \\
\hline & \multicolumn{3}{|c|}{$\begin{array}{l}\text { Control familiar } \\
(\mathrm{n}=64)\end{array}$} & \multicolumn{3}{|c|}{$\begin{array}{l}\text { No control familiar } \\
(\mathrm{n}=56)\end{array}$} & \multirow{2}{*}{$\begin{array}{l}\text { U Mann Whitney } \\
1772,5\end{array}$} \\
\hline ÍNDICE GLOBAL & 0,808 & 0,830 & 60,20 & 0,810 & 0,817 & 60,85 & \\
\hline - PERFIL & 0,847 & 1 & 60,93 & 0,792 & 1 & 60,01 & 1764,5 \\
\hline - ECONÓMICO & 0,715 & 0,833 & 63,73 & 0,627 & 0,833 & 56,81 & 1585,5 \\
\hline - MEDIOAMBIENTE & 0,610 & 0,617 & 61,30 & 0,581 & 0,583 & 59,59 & 1741 \\
\hline - SOCIAL & 0,626 & 0,647 & 63,23 & 0,564 & 0 & 57,38 & 1617,5 \\
\hline - Laboral y ética & 0,726 & 0,817 & 64,06 & 0,659 & 0 & 56,43 & 1564 \\
\hline - Derechos humanos & 0,674 & 0,750 & 66,04 & 0,550 & 0 & 54,17 & $13437,5 \dagger$ \\
\hline Sociedad & 0,580 & 0,600 & 61,56 & 0,524 & 0 & 59,29 & 1724 \\
\hline - Resp. productos & 0,545 & 0,578 & 60,17 & 0,552 & 0 & 60,88 & 1771 \\
\hline \multicolumn{8}{|c|}{ Panel B: Generación de la familia en la propiedad de la empresa } \\
\hline & \multicolumn{3}{|c|}{$\begin{array}{l}\text { Empresa familiar } \\
\text { fundador }(n=83)\end{array}$} & \multicolumn{3}{|c|}{$\begin{array}{l}\text { Empresa familiar no fundador } \\
(\mathrm{n}=37)\end{array}$} & U Mann Whitney \\
\hline ÍNDICE GLOBAL & 0,813 & 0,839 & 62,79 & 0,801 & 0,795 & 55,36 & 1345,5 \\
\hline - PERFIL & 0,795 & 1 & 57,49 & 0,880 & 1 & 67,26 & 1285,5 \\
\hline - ECONÓMICO & 0,650 & 0,833 & 59,31 & 0,728 & 0,833 & 63,16 & 1437 \\
\hline - MEDIOAMBIENTE & 0,588 & 0,600 & 59,92 & 0,615 & 0,633 & 61,81 & 1487 \\
\hline - SOCIAL & 0,604 & 0,676 & 63,04 & 0,580 & 0,588 & 54,80 & 1324,5 \\
\hline - Laboral y ética & 0,694 & 0,750 & 62,60 & 0,696 & 0,750 & 55,78 & 1361 \\
\hline - Derechos humanos & 0,569 & 0,750 & 56,91 & 0,721 & 0,750 & 68,55 & $1237,5 \dagger$ \\
\hline - Sociedad & 0,551 & 0,600 & 61,95 & 0,561 & 0,600 & 57,24 & 1415 \\
\hline - Resp. productos & 0,556 & 0,667 & 61,58 & 0,525 & 0,444 & 58,12 & 1447,5 \\
\hline
\end{tabular}

$\mathrm{n}=120 ; \dagger \mathrm{p}<0,10 ; * \mathrm{p}<0,05 ; * * \mathrm{p}<0,01 ; * * * \mathrm{p}<0,001$

INDICE GLOBAL es un índice total en transparencia informativa en RSC construido siguiendo las recomendaciones establecidas por el GRI a partir de la información que ofrece cada empresa en las variables definidas en las cuatro dimensiones consideradas en el GRI: perfil, económica, ambiental y social. Por control familiar entendemos la presencia del grupo propietario familiar en puestos como el presidente del consejo de administración o el máximo ejecutivo de la empresa. Por la generación de la familia consideramos si el fundador se encuentra en el grupo que ostenta la propiedad de la empresa o no.

gestión, implica una menor exigencia comunicativa hacia los stakeholders, dado que los dos grupos de interés más poderosos (directivos y accionistas) poseen una misma identidad.

Por lo que se refiere a la presencia del fundador en la empresa, los resultados muestran que este hecho no parece influir de manera significativa en el compromiso con la RSC de las empresas familiares (Tabla 2, Panel B).

En último lugar, nos planteamos si existen diferencias entre las empresas de propiedad familiar y no familiar, y a su vez dentro de las familiares según haya o no control familiar y esté presente el fundador o no, al medir la transparencia en RSC utilizando un índice de transparencia global, y los distintos sub-índices a partir de las recomendaciones del GRI (perfil, económico, ambiental y social).

En este sentido, en línea con los resultados comentados anteriormente, y acorde con la información proporcionada por los valores medianos y los rangos promedios, parece que tanto el índice global como las distintas categorías consideradas alcanzan valores superiores en la muestra de las empresas no familiares. Además, los resultados obtenidos tras aplicar la U de Mann Whitney a partir de los rangos promedios permiten concluir que las diferencias observadas son estadísticamente relevantes en todos los casos $\mathrm{y}$, por ende, no atribuibles al azar (Tabla 3). Sin embargo, los resultados no apoyan la existen cia de diferencias significativas dentro de las empresas familiares considerando un criterio de control o la generación familiar presente en la empresa para el caso del índice de transparencia en RSC (Tabla 4). Estos resultados sugieren así que el control familiar y la presencia de los fundadores no influyen en la amplitud de la transparencia en RSC.

\section{Conclusiones}

En los últimos años, la RSC se ha convertido en una práctica que, con relativa frecuencia, las empresas están utilizando de modo voluntario con la finalidad de mejorar las condiciones sociales y medioambientales en sus negocios, su reputación así como su relación con los distintos stakeholders. Así, 
distintos estudios longitudinales sobre información social corporativa ponen de manifiesto también un incremento paulatino del número de compañías que publican información sobre las acciones de RSC que desarrollan y de su contenido informativo (Deegan y Gordon, 1996; Moneva y Llena, 2000; Archel, 2003).

No son muchos los estudios que han analizado la influencia de la naturaleza familiar de una empresa sobre el compromiso en RSC ni sobre su transparencia informativa relacionada con la RSC. Nuestro trabajo pretende contribuir a esta línea de investigación analizando para una muestra de empresas españolas cotizadas en el periodo 20042010, en qué medida una de las principales tipologías de accionistas en nuestro país, la propiedad familiar así como si la presencia del fundador y de la familia en los órganos de control de la empresa, influye en la transparencia sobre las acciones de RSC llevadas a cabo por las empresas, así como con la cantidad de información divulgada. En línea con los estudios de Ndemanga y Koffi (2009) para Suecia y de López-Iturriaga y López de Foronda (2011) y Dam y Scholtens (2012) para el mercado europeo, encontramos un menor compromiso de las empresas con propiedad familiar con la publicación de información de RSC y con la cantidad de información publicada. Asimismo, nuestros resultados sugieren que no sólo la propiedad sino también el control podría influir en la decisión de publicar información por parte de las compañías familiares, ya que éstas publican en menor medida memorias o documentos de RSC si la familia tiene además el control de la gestión empresarial. Sin embargo, el control familiar no parece tener influencia en la cantidad de información divulgada medida a través de distintos índices de transparencia informativa en RSC. Por su parte, la presencia del fundador no influye ni en la publicación de información de RSC ni en la cantidad de información publicada.

Somos conscientes que la realización del estudio en un único país puede ser considerada como una limitación del mismo, pero ello permite superar los problemas asociados a muestras de varios países centradas en la mayoría de las ocasiones en las empresas de mayor tamaño. De igual forma, el análisis de la influencia de las variables familiares junto con otras relativas a la empresa que pueden influir en la transparencia informativa (como por ejemplo, su tamaño, sector, etc.) así como la utilización de otras metodologías no de naturaleza tan descriptiva como, por ejemplo, un análisis de regresión lineal o modelos de variable dependiente discreta, añadiría también valor a futuras investigaciones. Finalmente, estudios futuros podrían también abordar en cadena la decisión de publicar información y el nivel de información y su relación con el control familiar.

\section{Bibliografía}

Agle, B.R., Mitchell, R.K., y Sonnenfeld, J.A. (1999). Who matters to CEOs? An investigation of stakeholder attributes and salience, corporate performance, and CEO values. Academy of Management Journal, 42(5), 507-525.

Aguinis, H., y Glavas, A. (2012). What we know and don't know about corporate social responsibility: A review and research agenda. Journal of Management, 38, 932-968.

Anderson, R.C., Mansi, S.A., y Reeb, D.M. (2003). Founding family ownership and the agency cost of debt. Journal of Financial Economics, 68, 263-285.

Anderson, R.C., y Reeb, D.M. (2003). Foundingfamily ownership and firm performance: Evidence from the S\&P 500. The Journal of Finance, 58(3), 1301-1328.

Archel, P. (2003). La divulgación de la información social y medioambiental de la gran empresa española en el período 1994-1998: Situación actual y perspectivas. Revista Española de Financiación y Contabilidad, 117, 571-601.

Arora, P., y Dharwadkar, R., 2011. Corporate governance and corporate social responsibility (CSR): The moderating roles of attainment discrepancy and organization slack. Corporate Governance: An International Review, 19(2), 136-152.

Bachiller, P., Giorgino, M.C., y Paternostro, S. (2013). Analysis of social performance and board of directors in family firms: Evidence from quoted Italian companies. En Smyrnios, K., Panikkos, P., y Goel, S. (Eds.), "2nd. Handbook of Research on family firms", capítulo 8, (pp. 82-102). UK: Edward Elgar Publishers.

Barnea, A., y Rubin, A. (2010). Corporate social responsibility as a conflict between shareholders. Journal of Business Ethics, 97(1), 71-86.

Bear, S., Rhaman, N., y Post, C. (2010). The impact of board diversity and gender composition on corporate social responsibility and firm reputation. Journal of Business Ethics, 97(2), 207-221.

Berrone, P., Cruz, C., Gómez-Mejía, L.R., y LarrazaKintana, M. (2010). Socioemotional wealth and corporate responses to institutional pressures: Do family-controlled firms pollute less?" Administrative Science Quarterly, 55, 82-113.

Blair, M. (1995). Ownership and control: Rethinking governance for the twenty-first century. Washington DC: The Brooking Institution. 
Brammer, S., y Pavelin, S. (2008). Factors influencing the quality of corporate environmental disclosure. Business Strategy and the Environment, 17, 120-136.

Bushee, B.J. (1998). The influence of institutional investors on myopic R\&D investment behavior. Accounting Review, 73(3), 305-333.

Campbell, S.K. (1974). Flaws and fallacies in statistical thinking. New Jersey: Prentice-Hall.

Carroll, A.B. (1991). The pyramid of corporate social responsibility: Toward the moral management of organizational stakeholders. Business Horizons, 34, 3948 .

Claessens, S., Djankov, S., y Lang, L. (2000). The separation of ownership and control in East Asian corporations. Journal of Financial Economics, 58, 81-112.

Claessens, S., Djankov, S., y Lang, L. (2002). Disentangling the incentive and entrenchment effects of large shareholdings. The Journal of Finance, 57, 27412772 .

Comisión Europea (2002). Green Book: Promoting a European framework for corporate social responsibility. http://europa.eu.int/comm./employment_social/socdial/csr/greenpaper.htm.

Consolandi, C., Nascenzi, P., y Jaiswal-Dale A. (2008). Ownership concentration and corporate social performance: An empirical evidence for European firms. Corporate Responsibility Research Conference 2008, Belfast.

Crane, A., McWilliams, A., Matten, D., Moon, J., y Siegel, D.S. (2008). The corporate social responsibility agenda. En: A. Crane, A. McWilliams, D. Matten, J. Moon y D.S. Siegel (Eds.), The Oxford Handbook of CSR. Oxford: Oxford University Press.

Cuervo, A. (2004). El gobierno de la empresa. Un problema de conflicto de intereses. En E. Bueno Campos (ed.), El gobierno de la empresa. En busca de la transparencia y la confianza (pp. 115-138). Madrid: Pirámide.

Dam, L., y Scholtens, B. (2012). Does ownership matter for corporate social responsibility? Corporate Governance: An International Review, 20(3), 233-252.

Deegan, C., y Gordon, B. (1996). A study of the environmental disclosure practices of Australian corporations. Accounting and Business Research, 26(3), pp. 187-199.

Déniz, M.C., y Cabrera, M.K. (2005). Corporate social responsibility and family business in Spain. Journal of Business Ethics, 56(1), 27-41.

Dikolli, S.S., Kulp, S.L., y Sedatole, K.L. (2009). Transient institutional ownership and CEO contracting. Accounting Review, 84(3), 737-770.

Faccio, M., y Lang, L. (2002). The ultimate ownership of Western European corporations. Journal of Financial
Economics, 65, 365-395.

Fernández-Sánchez, J.L., Luna, L., y Baraibar, E. (2011). The relationship between corporate governance and corporate social behavior: A structural equation model analysis. Corporate Social Responsibility and Environmental Management, 18, 91-101.

Freeman, R.E. (1984). Strategic management: A stakeholder approach. Boston: Pitman Publishing Inc.

Freund, J., y Simon, G. (1994). Estadística elemental. México: Prentice-Hall Hispanoamericana.

Ghazali, N. (2007). Ownership structure and corporate social responsibility disclosure: Some Malaysian evidence. Corporate Governance, 7(3), 251-266.

Godos-Díez, J.L., Fernández-Gago, R., y CabezaGarcía, L. (2012). Propiedad y control en la puesta en práctica de la RSC. Cuadernos de Economía y Dirección de la Empresa, 15(1), 1-11.

Gómez-Mejía, L.R., Takács Haynes, K., NúñezNickel, M., Jacobson, KJL., y Moyano-Fuentes, J. (2007). Socioemotional wealth and business risks in familycontrolled firms: Evidence from Spanish olive oil mills. Administrative Science Quarterly, 52, 106-137.

Graafland, J.J. (2002). Corporate social responsibility and family business. Paper presented at the Research Forum of the Family Business Network 13th Annual Conference. Helsinki, Finland.

Graves, S.B., y Waddock, S.A. (1994). Institutional owners and corporate social performance. Academy of Management Journal, 37(4), 1034-1046.

Griffin, J.J., y Mahon, J.F. (1997). The corporate social performance and corporate financial performance debate: Twenty-five years of incomparable research. Business and Society, 36, 5-31.

Habbershon, T., Williams, M., y Macmillan, IC. (2003). A unified systems perspective of family firm performance. Journal of Business Venturing, 18, 451-465.

Haniffa, R.M., y Cooke, T.E. (2005). The impact of culture and governance on corporate social reporting. Journal of Accounting and Public Policy, 24, 391-430.

Harjoto, M.A., y Jo, H. (2011). Corporate governance and CSR nexus. Journal of Business Ethics, 100(1), 4567.

Hoopes, D.G., y Miller, D. (2006). Ownership preferences, competitive heterogeneity, and familycontrolled businesses. Family Business Review, 19(2), 89-101.

Johnson, RA., y Greening, D.W. (1999). The effects of corporate governance and institutional ownership types on corporate social performance. Academy of Management Journal, 42(5), 564-576.

Johnson, S., La Porta, R., López de Silanes, F., y Shleifer, A. (2000).Tunneling. American Economic Review, 90(2), 22-27. 
Jo, H., y Harjoto, M.A. (2011). Corporate governance and firm value: The impact of corporate social responsibility. Journal of Business Ethics, 103, 351-383.

Kuo, L., Yeh, CH., y Yu, H. (2012). Disclosure of corporate social responsibility and environmental management: Evidence from China. Corporate Social Responsibility and Environmental Management, 19, 273287.

La Porta, R., Lopez-de-Silanes, F., y Shleifer, A. (1999). Corporate ownership around the world. The Journal of Finance, 54, 471-517.

Lindgreen, A., Swaen, V., y Johnston, W. (2009). Corporate social responsibility: An empirical investigation of U.S. organizations. Journal of Business Ethics, 85(2), 303-323.

López-Iturriaga, F., y López-de-Foronda, O. (2011). Corporate social responsibility and reference shareholders: An analysis of European multinational firms. Transnational Corporations Review, 3(3), 1-11.

Mahapatra, S. (1984). Investor reaction to a corporate social accounting. Journal of Business Finance and Accounting, 11, 29-40.

McGuire, J., Dow, S., y Ibrahim, B. (2012). All in the family? Social performance and corporate governance in the family firm. Journal of Business Research, 65, 16431650 .

Mcvey, H., y Draho, J. (2005). U.S. family-run companies - They may be better than you think. Journal of Applied Corporate Finance, 17(4), 134-143.

Miller, D., y Le Breton-Miller, I. (2006). Family governance and firm performance: Agency, stewardship, and capabilities. Family Business Review, 19, 73-87.

Mitchell, R.K., Agle, B.R., y Wood, D.J. (1997). Toward a theory of stakeholder identification and salience: Defining the principle of whom and what really counts. Academy of Management Review, 22, 853-886.

Monks, R., y Minow, N. (1995). Corporate governance. Cambridge, MA: Blackwell.

Moneva, J.M., y Llena, F. (2000). Environmental disclosures in the annual reports of large companies in Spain. European Accounting Review, 9(1), 7-29.

Moore, G. (2001). Corporate social and financial performance: An investigation in the U.K. supermarket industry. Journal of Business Ethics, 34, 299-315.

Ndemanga, DA., y Koffi, ET. (2009). Ownership structure, industry sector and corporate social responsibility (CSR) practices: - The case of Swedish listed companies. Master of Science in Accounting, Master Degree Project No. 2009:31.

Orlitzky, M., Schmidt, F.L., y Rynes, S.L. (2003).
Corporate social and financial performance: A metaanalysis. Organization Studies, 24, 403-441.

Ortiz de Mandojana, N., Aragón, A., y Delgado, J. (2011). La relación entre la propiedad institucional y de los directivos y el desempeño medioambiental. Cuadernos de Economía y Dirección de Empresa, 14, 222-230.

Peloza, J. (2009). The challenge of measuring financial impacts from investments in corporate social performance. Journal of Management, 35, 1518-1541.

Porter, M.E. (1992). Capital choices: Changing the way America Invests in Industry. Journal of Applied Corporate Finance, 5(2), 4-16.

Prado-Lorenzo, J.M., Gallego-Álvarez, I., y GarcíaSánchez, I.M. (2009). "Stakeholder engagement and corporate social responsibility reporting: The ownership structure effect". Corporate Social Responsibility and Environmental Management, 16(2), 94-107.

Sharma, P. (2008). Commentary, familiness: Capital stocks and flows between family and business. Entrepreneurship: Theory and Practice, 32, 971-977.

Sharma, P., Chrisman, J.J., y Chua, J.H. (1997). Strategic management of the family business: Past research and future challenges. Family Business Review, 10, 1-35.

Sharma, P., Chrisman, J.J., y Gersick, K.E. (2012). 25 years of Family Business Review: Reflections on the past and perspectives for the future. Family Business Review, 25(5), 5-15.

Simpson, W.G., y Kohers, T. (2002). The link between corporate social and financial performance: Evidence from the banking industry. Journal of Business Ethics, 35, 97-109.

Schulze, W.S., Lubatkin, M.H., y Dino, R.N. (2003). Toward a theory of agency and altruism in family firms. Journal of Business Venturing, 8, 473-450.

Testera, A., y Cabeza, L. (2013). Análisis de los factores determinantes de la transparencia en RSC en las empresas españolas cotizadas. Intangible Capital, 9(1), 225-261.

Yong, W., Kyun, Y., y Martynov, A. (2011). The effect of ownership structure on corporate social responsibility: Empirical evidence from Korea. Journal of Business Ethics, 104, 283-297.

Wallace, R., Naser, K., y Mora, A. (1994). The relationship between the comprehensiveness of corporate annual reports and firm characteristics in Spain. Accounting and Business Research, 25(97), 41-53.

Walls, J., Berrone, P., y Phan, P. (2012). Corporate governance and environmental performance: Is there really a link? Strategic Management Journal, 33, 885-913. 